\title{
Effect of Interpregnancy Interval on Adverse Maternal and Perinatal Outcomes at Katutura Regional Referral Hospital in Namibia: A Retrospective Cohort Study, 2005 to 2015
}

\section{Lyasimana Lithaneninn Ndaninginan ${ }^{1,3}$, Pendo Mlay ${ }^{1}$, Raziya Gaffur ${ }^{1}$, Benjamin Joseph Nggada $^{3}$ and Michael Johnson Mahande ${ }^{2 *}$}

${ }^{1}$ Department of Obstetrics and Gynecology, Kilimanjaro Christian Medical Center, Moshi, Tanzania

${ }^{2}$ Department of Epidemiology and Biostatistics, Institute of Public Health, Kilimanjaro Christian Medical University College, Moshi, Tanzania

${ }^{3}$ Department of Obstetrics and Gynecology,Katutura Referral Regional Hospital, Windhoek, Namibia

*Corresponding Author: Michael Johnson Mahande, Department of Epidemiology and Biostatistics, Institute of Public Health, Kilimanjaro Christian Medical University College, Moshi, Tanzania.
Received: September 05, 2020

Published: November 18, 2020

(C) All rights are reserved by Michael Johnson Mahande., et al.

\begin{abstract}
Objective: To determine the effect of interpregnancy interval (IPI) on adverse and perinatal outcomes among Namibian women who delivered at Katutura regional referral Hospital from January 2005 to December 2015.

Methods: Retrospective cohort study conducted at Katutura regional referral hospital Windhoek in Namibia. A total of 3219 women who had two consecutive singleton deliveries from 2005 - 2015 were studied. The outcome of interest were maternal outcomes (preeclampsia, anemia and maternal death) and perinatal outcomes (preterm birth, low birth weight, neonatal intensive care unit and perinatal death).

Data were analyzed using STATA version 15. A log-binomial regression model was used to estimate the relative risk for adverse outcomes with their respective $95 \%$ confidence intervals (CIs). A robust variance estimator was used to take into account for repeated observations/births from the same mother.

Results: Nearly half (52.9\%) of the women had optimal interpregnancy interval (24 - 59 months). Women who conceived within a short IPI had increased risk of preeclampsia in their next pregnancy (ARR 2.70.95\% CI 1.55 - 4.71). Both short and long IPI were associated with an increased risk of anemia (ARR 2.76; 95\% 1.59 - 3.94) and (ARR 2.51; 95\% 1.38 - 4.57) respectively. Short IPI was associated with 3\% maternal death. Short IPI was associated with increased risk of preterm birth (ARR1.67; $95 \% 1.36-2.06$ ) and low birth weight (ARR1.34; 95\% 1.03 - 1.74), respectively.

Conclusion: We confirm that both short and long IPI were associated with adverse maternal and perinatal outcomes. Our finding suggest a need for a targeted intervention such as modern contraceptive use to promote optimal IPI.
\end{abstract}

Keywords: Interpregnancy Interval; Adverse Maternal Outcomes; Adverse Perinatal Outcomes; Namibia

\section{Introduction}

Interpregnancy interval (IPI) is defined as the time period between the delivery of previous live birth and conception of the next pregnancy [1]. Categorization for short and long IPI have not been standardized. A short IPI has been defined as less than 3, 6, 9, 12, or $<24$ months, while long IPI is usually defined as an IPI more than $\geq 60$ months $[2,3]$. In 2005 , the world health organization
(WHO) recommended an interval of at least 24 months after a live birth before attempting the next pregnancy to reduce the risk of adverse maternal, perinatal and infant outcomes [4].

In this study IPI has been categorized into short/sub optimal ( $<24$ months), optimal (24 - 59 months) and long ( $\geq 60$ months) intervals. Both short and long inter-pregnancy interval have been 
Effect of Interpregnancy Interval on Adverse Maternal and Perinatal Outcomes at Katutura Regional Referral Hospital in Namibia: A Retrospective Cohort Study, 2005 to 2015

associated with an increased risk of adverse pregnancy outcomes [5-7], while optimal IPI has been associated with lower risk of adverse maternal and perinatal outcomes $[3,4,7,8]$.

The effect of long interpregnancy interval (LIPI) and short interpregnancy interval (SIPI) on maternal and perinatal outcomes has been supported by two hypothesis [5,9]. First, maternal depletion hypothesis, this states that the short time interval between pregnancy doesn't allow recovery of micronutrients needed for the next pregnancy, thus the reason why the SIPI is associated with more adverse pregnancy outcome as compared to LIPI $[5,9,10]$. Second, physiological regression hypothesis, which states that the benefit associated with previous pregnancy gradually lost as the duration between pregnancies increases and thereafter the mother returns to primigravida state [10]. Together with extreme IPI (SIPI and LIPI), there are other factors which have been associated with maternal and perinatal adverse outcomes in the subsequent pregnancy, such as genetics, comorbidities, socio-demographics factors, lifestyle and socioeconomic status [5,9].

Previous studies have shown that, family planning and use of modern contraceptive methods is an effective measure to optimize IPI [11-13]. However, modern contraceptive use, especially postpartum contraceptives has not been fully utilized in most low resourced countries which resulted in majority of women being at increased risk of extreme IPI [11,14].

Previous studies in Tanzania have reported that majority of women experience sub-optimal IPI ranging from $33.9 \%$ to $52 \%$ $[3,15,16]$. These studies also demonstrated that SIPI $(<24$ months $)$ was associate with an increased risk of adverse maternal (maternal anemia, placenta previa, uterine rupture) and perinatal (preterm birth, low birth weight admission to neonatal intensive care and congenital malformations) outcomes. LIPI ( $\geq 60$ months) has also been associated with an increased risk of preeclampsia/eclampsia, hemorrhage, preterm delivery and low birth weight $[3,16]$. All the named outcomes have been reported as the common cause of maternal and perinatal morbidity and mortality in low resource settings which include Namibia [7].

In Namibia, neonatal mortality stands at a rate of 19 per 1000 live birth, of which $54 \%$ of the fatalities are attributable to premature birth [17]. The maternal mortality also is high as 265/100 000 live birth in 2015 [18]. The common causes of maternal and child mortality include postpartum hemorrhage, eclampsia, preterm birth and low birth weight [19]. All these conditions have been linked with sub-optimal IPI in the previous studies. There is scant information on the effect of IPI on adverse pregnancy outcomes in Namibia. Understanding the effect of IPI on adverse maternal and perinatal outcomes will enable implementation of measures directed to reduce maternal and perinatal mortality and morbidity.

\section{Aim of the Study}

This study aimed to determine the effect of IPI on adverse maternal and perinatal outcomes among women who delivered at Katutura regional referral hospital from January 2005 to December 2015 .

\section{Materials and Methods}

Study design and setting

A retrospective cohort study was conducted using maternallylinked data from Katutura regional referral hospital birth register between 2005 and 2015. Katutura hospital is a regional referral and teaching hospital that serves the densely populated Khomas region, with a population of 342.141 (Namibian National Census, 2011). It receives referrals for the Khomas region as well as from other thirteen regions. Pregnant women categorized as having complications and high risk pregnancies are referred to Katutura regional referral state hospital (KSH) for further management. $\mathrm{KSH}$ has 840 bed capacity, located in Windhoek in the capital city of Namibia. The maternity unity performs approximately 4000 - 6000 deliveries annually and offers comprehensive obstetric services to the population it serves. The main catchment area of Katutura hospital is Khomas region.

\section{Study population}

All women who had delivered two consecutive singleton, in KSH between January 2005 and December 2015. The study included all mothers who had delivered two or more consecutive singleton infants, at KSH during the study period. All women with twin deliveries, diabetes mellitus, antiphospholipid syndrome, cervical insufficiency, thyroid disease, chromosomal abnormality, multiple pregnancy and those with missing information were excluded.

\section{Sampling and design, sample and sampling}

A total of 37, 435 deliveries were recorded between 2005 and 2015. Of these, 3219 were eligible for this study and constituted the cohort. These women were followed retrospectively through their medical records. We restricted our analysis to two consecutive singleton deliveries of the same mother that occurred at $\mathrm{KSH}$ during the study period. Women who had comorbidities, an incomplete record, only one delivery or who had two but not consecutive singleton deliveries recorded in KSH birth register during the study period were excluded in the analysis (Figure 1). 


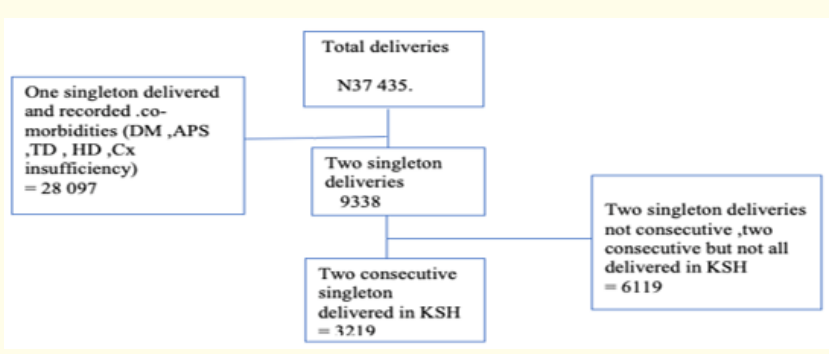

Figure 1: Schematic diagram of the study participants $(\mathrm{N}=3219)$.

\section{Study variables}

The outcome of interest include adverse maternal and perinatal outcomes. The adverse maternal outcomes include maternal anemia, preeclampsia and maternal death while perinatal outcomes include low birth weight, preterm birth, use of neonatal intensive care unit and neonatal death. A woman was considered to have anemia if at any point during pregnancy she had Hemoglobin level below 10.5 grams per deciliter $(10.5 \mathrm{~g} / \mathrm{dl})$. Preeclampsia was defined as a progressive disorder characterized by development of hypertension $140 / 90 \mathrm{mmHg}$ or more with or without proteinuria after the $20^{\text {th }}$ week of gestation in a previously normotensive and non-proteinuric woman.

Interpregnancy interval (IPI) was the main exposure of interest and it was computed as follows:

IPI (days) $=[$ Date of birth of index child - Date of birth of preceding child] - Gestation age at birth of index child.

IPI was originally recorded as a continuous variable, then it was categorized into < 24 months (short IPI), 24 - 59 (optimal IPI) and ( $\geq 60$ months) (long IPI). Other covariates include maternal age at birth, residential area, occupation, marital status, education level.

\section{Data collection methods}

Data extraction sheet was used to obtain all relevant information from demographic characteristic and immediate maternal and neonatal complication. A structured questionnaire was used to collect information from patient file. All relevant information about study variables was extracted from the patients' file using a wellstructured English extraction sheet. This was then be transferred into a computer database containing all corresponding variables needed for the study.

Information for all deliveries that occur at the KSH department of Obstetrics and Gynecology are prospectively collected and entered in a computerized database system in the ward birth register by a data clerk. Data on pregnancy outcomes were extracted from archived medical record. Neonates who are admitted into the neonatal care unit are recorded in the neonatal registry and each discharged neonatal record is stored with the mother's file. Licensed health professionals, namely midwife nurses and medical doctors' conducts and record maternal assessments from admission to discharge using a standardized booklet for all women who deliver at $\mathrm{KSH}$. Information on the neonates admitted to the neonatal care unit is recorded in neonatal registry and the file that have the maternal identification number. Verbal consent is obtained from and for each mothers on admission prior to the conduct of any research activity utilizing her medical record in according with the principals of Good Practice in conduct of Human Subjects Research. The birth register has unique identification numbers.

\section{Data analysis}

Data were analyzed using STATA version 15 (Stata Corp., Texas and US). Descriptive statistics were summarized using mean standard deviation while Chi square statistics was used to determine the association between interpregnancy interval and outcomes of interest in bivariate analysis. A log-binomial regression model was used to estimate the relative risk with $95 \%$ confidence intervals (CIs) for adverse outcomes associated with IPI while adjusting for potential confounding variables.

A p-value of less than $5 \%$ was considered statistically significant. We used mothers as the primary unit of analysis. The clustered analysis technique was employed, where births of the same mother formed a cluster. A variable was considered to be a confounder if the inclusion of a variable in a model changed the crude estimate to adjusted estimate by $\geq 10 \%$. Generalized Additive Models were used to determine if there was a dose-response relationship between IPI and adverse maternal and perinatal outcomes.

\section{Ethical considerations}

Ethical clearance certificates No 2298 were obtained from Kilimanjaro Christian Medical University College Research Ethical Committee in Tanzania and the Ministry of Health and Social Services (MOHSS) in Namibia prior to the commencement of this study. The permission to carry out the study using the KSH birth register data was granted from the hospital medical superintendent. The requirement for maintaining the confidentiality of study participant information was adhered by the use of a unique identification number for each research participant.

\section{Results}

Distribution of inter pregnancy interval

More than half (52.9\%) of the women had optimal IPI (24 - 59 months). Almost $27 \%$ of participant had long IPI ( $\geq 60$ months) (Table 1). 
Effect of Interpregnancy Interval on Adverse Maternal and Perinatal Outcomes at Katutura Regional Referral Hospital in Namibia: A Retrospective Cohort Study, 2005 to 2015

\begin{tabular}{|l|c|}
\hline $\begin{array}{l}\text { Inter pregnancy } \\
\text { interval (months) }\end{array}$ & $\mathbf{n ~ ( \% ) ~}$ \\
\hline$<11$ & $90(2.80)$ \\
\hline $11-17$ & $197(6.12)$ \\
\hline $18-23$ & $371(11.53)$ \\
\hline $24-59$ & $1705(52.97)$ \\
\hline$\geq 60$ & $856(26.59)$ \\
\hline
\end{tabular}

Socio-demographic characteristic of study participants

A total of 3219 deliveries were analyzed. Majority (73.55\%) were aged 20 - 34 years. Majority (76\%) of the participants had secondary education and conceived within the short IPI of 18 - 23 months. Among those who conceived during this interval, majority $(71 \%)$ were not in marital union and $70 \%$ did not use contraceptives (Table 2).

Table 1: Distribution of inter pregnancy interval (months)

$$
(\mathrm{N}=3,219) \text {. }
$$

\begin{tabular}{|c|c|c|c|c|c|}
\hline & $<11$ & $11-17$ & $18-23$ & $24-59$ & $\geq 60$ \\
\hline Maternal Age (years)* & $26.6(4.13)$ & $26.42(6.70)$ & $25(6.35)$ & $23.90(5.47)$ & $24.56(5.05)$ \\
\hline \multicolumn{6}{|l|}{ Age categories (years) } \\
\hline$\leq 19$ & $2(2.2)$ & $41(20.81)$ & $74(19.95)$ & 380 (22.29) & $139(16.24)$ \\
\hline $20-34$ & $83(92.22)$ & $137(69.54)$ & 263 (70.89) & 1254 (73.55) & $702(82.01)$ \\
\hline$\geq 35$ & $5(5.56)$ & $19(9.64)$ & $34(9.16)$ & $71(4.16)$ & $15(1.75)$ \\
\hline \multicolumn{6}{|l|}{ Mother's education } \\
\hline Primary & $15(16.67)$ & $25(12.69)$ & $61(16.44)$ & $206(12.08)$ & $59(6.89)$ \\
\hline Secondary & $56(62.22)$ & $138(70.05)$ & $282(76.01)$ & $1035(60.70)$ & $552(64.49)$ \\
\hline College and University & $19(21.11)$ & $34(17.26)$ & $28(7.55)$ & $464(27.21)$ & $245(28.62)$ \\
\hline \multicolumn{6}{|l|}{ Mother's occupation } \\
\hline Unemployed & $40(44.44)$ & $85(43.15)$ & $186(50.13)$ & 847 (49.68) & 419 (48.95) \\
\hline Employed & $50(55.56)$ & $112(56.85)$ & 185 (49.87) & $858(50.32)$ & 437 (51.05) \\
\hline \multicolumn{6}{|l|}{ Religion } \\
\hline Catholic & $27(30)$ & $55(27.92)$ & $123(33.15)$ & 388 (22.76) & $190(22.20)$ \\
\hline Lutheran & $46(51.11)$ & $110(55.84)$ & $148(39.89)$ & $810(47.51)$ & $412(48.13)$ \\
\hline Other & 17 (18.89) & $32(16.24)$ & $100(26.95)$ & $507(29.74)$ & $254(29.67)$ \\
\hline \multicolumn{6}{|l|}{ Residence } \\
\hline Informal Settlement & $16(17.78)$ & $39(19.80)$ & $67(18.06)$ & $371(21.76)$ & $211(24.65)$ \\
\hline Rural & 8 (8.89) & $25(12.69)$ & $29(7.82)$ & $147(8.62)$ & $36(4.21)$ \\
\hline Urban & $66(73.33)$ & $133(67.51)$ & $275(74.12)$ & $1187(69.62)$ & $609(71.14)$ \\
\hline \multicolumn{6}{|l|}{ Marital status } \\
\hline Not-in-union & $63(70.00)$ & $134(68.02)$ & $265(71.43)$ & 1067 (62.58) & $591(69.04)$ \\
\hline In-union & $27(30.00)$ & $63(31.98)$ & $106(28.57)$ & $638(37.42)$ & $265(30.96)$ \\
\hline \multicolumn{6}{|l|}{ Family Planning Use } \\
\hline No & $64(71.11)$ & $148(75.13)$ & $259(69.81)$ & 1299 (76.19) & $632(73.83)$ \\
\hline Yes & $26(28.89)$ & 49 (24.87) & 112 (30.19) & $406(23.81)$ & $224(26.17)$ \\
\hline \multicolumn{6}{|l|}{ Parity } \\
\hline $1-3$ & $79(87.78)$ & $172(87.31)$ & $314(84.64)$ & $1541(90.38)$ & 771 (90.07) \\
\hline$\geq 4$ & $10(11.11)$ & $25(12.69)$ & $57(15.36)$ & $164(9.62)$ & $85(9.93)$ \\
\hline
\end{tabular}

Table 2: Sociodemographic characteristic of study participants by IPI at baseline birth (N = 3219).

*Mean (Standard Deviation). 
Distribution of maternal outcomes by interpregnancy interval

The proportion of preeclampsia was more prevalent than anemia across all IPI categories (Figure 2). The risk of preeclampsia was higher among women with long IPI ( $\geq 60$ months) compared to optimal IPI (24 - 59 months) (2.8\% vs. $1.9 \%$, respectively) while the risk of anemia was higher among women with short IPI (18 - 23 months) compared to optimal IPI (1.35\% vs. $1.06 \%)$

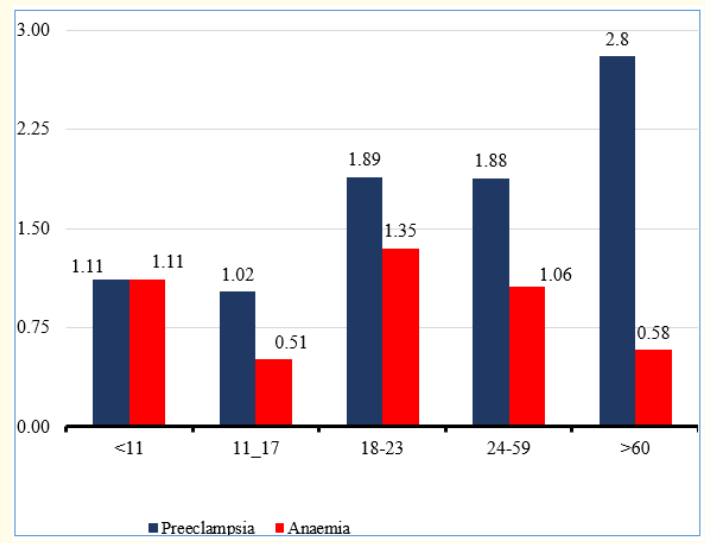

Figure 2: Proportion of maternal outcomes among the study participants by IPI $(\mathrm{N}=3219)$.

\section{Distribution of perinatal outcomes by IPI categories}

The short IPI was associated with adverse perinatal outcomes of interest. The risk of preterm birth was $22.2 \%$ among women with an IPI of less than 11 months. Women who had an IPI of 11

- 17 months had also an increased risk of experiencing low birth weight delivery and perinatal death (20.8\% vs $3.09 \%$, respectively) (Figure 3).

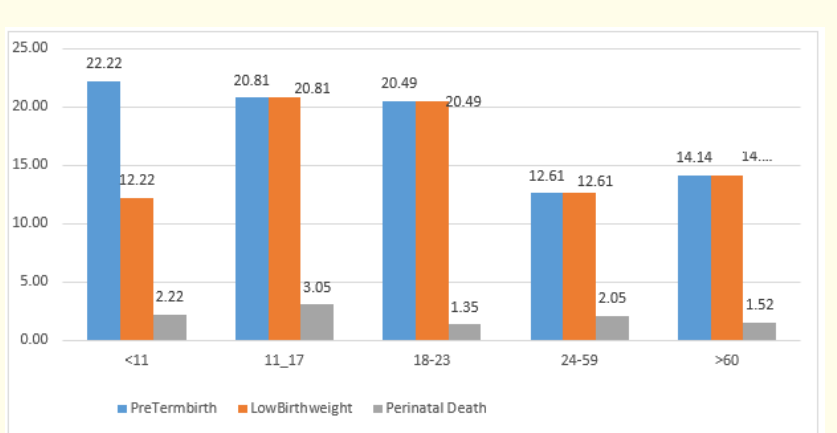

Figure 3: Distribution of perinatal outcomes among the study participants by IPI categories $(\mathrm{N}=3,219)$.

Effect of the IPI on adverse maternal outcomes in subsequent pregnancy

The effect of the IPI on adverse maternal outcomes in subsequent pregnancy has been displayed in figure 4 . Women who conceived within an interval of 18 - 23 months after their previous pregnancy had nearly three times (ARR 2.70; 95\% CI: 1.55 - 4.71) higher risk of developing preeclampsia in their next pregnancy compared with those who had optimal IPI (24 - 59 moths). The risk of having anemia in the subsequent pregnancy was also nearly 3-fold among women who conceived within an IPI of 11 - 17 months (ARR 2.76; $95 \%$ CI: 1.59 - 3.94) and those who conceived within an IPI of $\geq 60$ months (ARR 2.51; 95\% 1.38 - 4.57) compared to their counterparts with optimal IPI. We also found that women who had an IPI of $<11$ months had 5\% (ARR 1.05; 95\% CI: 1.04 - 1.05) increased risk dying in their subsequent pregnancy due to pregnancy related complications as compared to their counterparts with optimal IPI. On the other hand, women who had long IPI ( $\geq 60$ months) had $3 \%$ (ARR 0.97; 95\% CI: 0.96 - 0.97) lower risk of dying due to pregnancy related complications in their subsequent pregnancy compared to their counterparts with optimal IPI. More than half (52.9\%) of the women had optimal IPI (24 - 59 months).

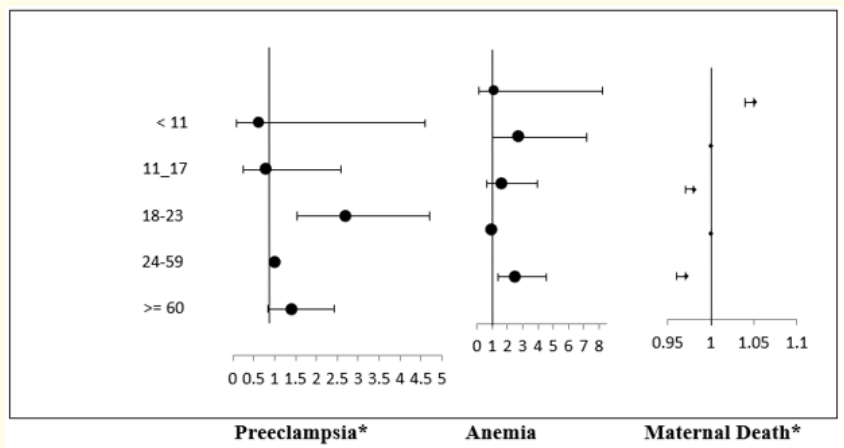

Figure 4: Effect of the IPI on adverse maternal outcomes in subsequent pregnancy $(n=3,219)$.

*ARR (Adjusted Relative Risk): Adjusted for maternal characteristics and obstetric outcomes in first pregnancy.

Effect of IPI on the risks of adverse perinatal outcomes

The effect of IPI on the risks of adverse perinatal outcomes has been shown in table 3. The risk of preterm birth (ARR1.67; 95\% CI: 1.36 - 2.06) and low birth weight (ARR1.34; 95\% CI: 1.03 - 1.74) in the subsequent pregnancy was higher among women with short IPI (11 - 17 months) compared with their counterparts who had optimal IPI. Babies who were born to mothers with long IPI (of $\geq$ 60 months) had 22\% (ARR 0.78; 95\% 0.60 - 1.00) low risk of being admitted in the NICU as compared to their counterparts who were born to mothers with an optimal IPI 24 - 59 months. 
Effect of Interpregnancy Interval on Adverse Maternal and Perinatal Outcomes at Katutura Regional Referral Hospital in Namibia: A Retrospective Cohort Study, 2005 to 2015

\begin{tabular}{|c|c|c|c|c|}
\hline & \multicolumn{4}{|c|}{ Perinatal Outcome [CRR $(95 \% \mathrm{CI})]$} \\
\hline & РTB & LBW & PM & NICU \\
\hline \multicolumn{5}{|c|}{ IPI quantiles (months) } \\
\hline$<11$ & $0.99(0.66-1.49)$ & $0.81(0.49-1.35)$ & $0.80(0.34-1.91)$ & $1.19(0.69-2.05)$ \\
\hline $11-17$ & $1.71(1.39-2.10)$ & $1.37(1.05-1.78)$ & $1.39(0.88-2.21)$ & $1.27(0.88-1.83)$ \\
\hline $18-23$ & $1.04(0.84-1.28)$ & $0.98(0.77-1.24)$ & $1.25(0.86-1.81)$ & $0.72(0.50-1.04)$ \\
\hline $24-59$ & Ref & Ref & Ref & Ref \\
\hline \multirow[t]{3}{*}{$\geq 60$} & $0.98(0.84-1.15)$ & $0.91(0.76-1.09)$ & $0.74(0.53-1.04)$ & $0.79(0.62-1.02)$ \\
\hline & \multicolumn{4}{|c|}{ Perinatal Outcome [ARR $\left.(95 \% \mathrm{CI})^{*}\right]$} \\
\hline & PTB & LBW & PM & NICU \\
\hline \multicolumn{5}{|c|}{ IPI quantiles (months) } \\
\hline$<11$ & $1.09(0.73-1.63)$ & $0.81(0.48-1.36)$ & $0.65(0.27-1.54)$ & $1.02(0.58-1.79)$ \\
\hline $11-17$ & $1.67(1.36-2.06)$ & $1.34(1.03-1.74)$ & $1.18(0.75-1.87)$ & $1.21(0.84-1.76)$ \\
\hline $18-23$ & $0.99(0.81-1.24)$ & $0.96(0.75-1.21)$ & $1.02(0.70-1.48)$ & $0.65(0.45-0.94)$ \\
\hline $24-59$ & Ref & & Ref & Ref \\
\hline$\geq 60$ & $1.01(0.86-1.18)$ & $0.92(0.77-1.11)$ & $0.73(0.52-1.02)$ & $0.78(0.60-1.00)$ \\
\hline
\end{tabular}

Table 3: Effect of IPI on the risks of adverse perinatal outcomes $(\mathrm{N}=3,219)$.

*ARR adjusted relative risk adjusted for maternal age, maternal education, religion, residence, marital status and parity. PTB: Preterm Birth; LBW: Low Birth Weight; PM: Perinatal Mortality; NICU: Neonatal Intensive Care Unit.

\section{Discussion}

Nearly half $(52.9 \%)$ of the women in the study population adhered to the World Health organization recommendation for optimal interpregnancy interval (24 - 59 months). Both extreme IPIs (SIPI and LIPI) were associated with adverse maternal and neonatal adverse outcomes. The short IPI was associated with an increased risks of preeclampsia, maternal anemia, maternal deaths, preterm birth and low birth weight in the subsequent pregnancy. On the other hand, long IPI was associated with an increased risk of maternal anemia. Our study shows a significant high percentage $(47.1 \%)$ of mother who fell in extreme IPI and this could be explained by the high number of mothers approximately 70 to $75 \%$ did not use any contraceptives methods as well as lifestyle, hence close interval between pregnancy and probably had unplanned pregnancy despite that modern contraceptive methods are available in all health facilities with no cost to the users.

The association of SIPI with adverse pregnancy outcomes in our study are in line with previous prospective cohort studies conducted in Tanzania $[3,16,20]$. Authors in these studies reported that women who had short IPI of less than 18 months had an increased risk of maternal anemia, preeclampsia and postpartum hemorrhage in their subsequent pregnancy as compared to women who had optimal IPI. Our find is also consistent with Brazilian study which reported an increased risk of maternal anemia and maternal death among mother who conceived within SIPI [8]. In contrast to our study, some authors did not found an association between SIPI with preeclampsia as well as long IPI with anemia [7,8,21]. The higher risk of adverse perinatal outcomes associated with SIPI noted in our study could possibly be explained by the maternal depletion hypothesis, poor socio-economic status and residual risks factors such as infections and other co-morbidities from previous pregnancies. Infection and co-morbidities have been associated with inflammatory processes that could affect the placental function, resulting in poor placental perfusion and the destabilization of fetal membrane integrity hence pre-term delivery and low birth-weight [22]. If the mother did not restore the micronutrients depleted by the previous pregnancy, especially iron, this leads to an increase risk of maternal anemia in the subsequent pregnancy, consequently impaired growth and development of the fetus, that eventually result in perinatal adverse outcomes [5]. Anemia during pregnancy may cause low birth weight, preterm birth, and perinatal, neonatal and maternal mortality because development of the fetus highly depend on oxygen and other nutrients delivery to the fetus, if the mother cannot supply adequate oxygen, this will compromise intra-uterine growth and development [23-25].

Regarding the association of SIPI with increased risk of preeclampsia among women with short IPI in our study population, could be attributed to young maternal age ( $\leq 20$ years), confirming that this age group is at high risk of obstetric complications that may results in adverse pregnancy outcomes because they are still 
in the process of growth [26]. Secondly, this can could be due to inadequate recovery from underlying risk factors such as delaying in clearing antiangiogenic factors such as soluble form like tyrosine kinase -1 (sflt -1) known to disturb the equilibrium in placenta angiogenesis result in preeclampsia/eclampsia that will eventually result in preterm birth or low birth weight $[22,27]$.

The association between LIPI and increased risk of anemia in our study, may be attributed to life style, poor social status compounded by effect of co-morbidities related to advance maternal age which might lead to anemia and its complications. Another explanation could be regression hypothesis, confirming that benefit acquired during previous pregnancy have vanish and maternal physiology have returned to nulliparous state, maternal body cannot meet the demand for iron and other micro-nutrients, hence anemia [5]. Advance maternal age comes with co-morbidities and their complications that could be the cause of anemia.

\section{Strength and Limitations}

This study used a large sample size and data from a longer period sufficient to improve the homogeneity of the data and give an adequate representation of the study population. To the best of our knowledge, this study is the first to assess the effect of both IPI and previous obstetric characteristics on subsequent pregnancy outcomes in Namibia.

Our study is limited by the small number of women with two successive deliveries during the study period. It is possible that the association between IPI and adverse maternal and perinatal outcomes is similar among the selected cohort of women with two consecutive deliveries. This study lacks information on some potential confounders such as infertility, sub-infertility and pregnancy loss. The effect of these factors to our finding was not quantified. We acknowledge that in retrospective study, the data utilized may have missing information, hence we were not able to adjust to risk factors such as co-morbidities and life style that could influence the pregnancy outcomes.

\section{Conclusion}

We confirm that extreme IPI were associated with adverse maternal and perinatal outcomes. Majority of adverse outcomes were associated with SIPI and interestingly SIPI was associated with increased risk of preeclampsia which was contrary to previous studies done elsewhere. It stands to reason therefore that this study provides critical information which can help health care providers to identify and give essential and targeted intervention relevant to improve maternal and fetal health, provide extensive counselling on postpartum contraceptives methods to attain optimal IPI and eventually improve pregnancy outcomes. Furthermore, a prospective study is necessary to discover which category of Namibian women have the greatest burden of adverse pregnancy outcomes in order to reduce maternal and perinatal mortality and attain the Sustainable Development Goal by 2030.

\section{Acknowledgments}

The department of obstetrics and gynecology in Katutura regional referral hospital is acknowledged for providing opportunity and availed the patient's files where the data extracted to conduct this study. Equally, the assistant researchers are acknowledged for their time and patience when they were collecting valuable information. Without them, the goal of the study would not be realized.

\section{Data Availability}

The data used to support the findings of this study are available from the corresponding author upon request.

\section{Author Contributions}

All authors contributed to the review and drafting of the study protocol. Data collection was done by local staff. Collected data were verified and analyzes by all authors. All authors contributed to the review and drafting of the paper and approved the final version.

\section{Conflicts of Interest}

The authors declare that they have no conflicts of interest.

\section{Bibliography}

1. Fareid S., et al. "Effect of Interpregnancy Interval on Pregnancy Outcome among Pregnant Women Attending Delivery at Belqas hospital". IOSR Journal of Nursing and Health Science 4.4 (2015): 5-13

2. Conde-Agudelo A., et al. "Effect of the interpregnancy interval on perinatal outcomes in Latin America". Obstetrics and Gynecology 106.2 (2005): 359-366.

3. Mahande MJ and Obure J. "Effect of interpregnancy interval on adverse pregnancy outcomes in northern Tanzania: a registrybased retrospective cohort study". BMC Pregnancy and Childbirth 16.1 (2016): 1-9.

4. World Health Organisation. Report of a WHO Technical Consultation on Birth Spacing (2007).

5. Kozuki N and Walker N. "Exploring the association between short/long preceding birth Intervals and child mortality: Using reference birth interval children of the same mother as comparison". BMC Public Health 13.3 (2013): S6. 
6. Gemmill A and Lindberg LD. "Short interpregnancy intervals in the United States”. Obstetrics and Gynecology 122.1 (2013): 64-71.

7. Hanley GE., et al. "Interpregnancy interval and adverse pregnancy outcomes an analysis of successive pregnancies". $O b$ stetrics and Gynecology 129.3 (2017): 408-415.

8. Conde-Agudelo A. "Maternal morbidity and mortality associated with interpregnancy interval: cross sectional study". British Medical Journal 321.7271 (2000): 1255-1259.

9. Conde-Agudelo A., et al. "Effect of Birth Spacing on Maternal, Perinatal, infant and Child Health: A systemic Review of Causal Mechanisms". American Journal of Obstetrics and Gynecology 192.2 (2012): 342-349.

10. Miller JE. "Birth intervals and perinatal health: an investigation of three hypotheses". Family Planning Perspectives 23.2 (1991): 62-70.

11. Towriss CA and Timæus IM. "Contraceptive use and lengthening birth intervals in rural and urban Eastern Africa”. Demographic Research 38.64 (2018): 2027-2052.

12. Yeakey MP., et al. "How Contraceptive Use Affects Birth Intervals: Results of a Literature Review". Studies in Family Planning 40.3 (2009): 205-214.

13. Bibi S., et al. "Postpartum contraception utilization and its impact on inter pregnancy interval among mothers accessing maternity services in the public sector hospital of Hyderabad Sindh". Pakistan Journal of Medical Sciences 35.6 (2019): 14821487.

14. Smith DP. "Breastfeeding, contraception, and birth intervals in developing countries". Studies in Family Planning 16.3 (1985): 154-163.

15. Exavery A., et al. "Levels and correlates of nonadherence to WHO recommended inter-birth intervals in Rufiji, Tanzania". BMC Pregnancy Childbirth 12 (2012): 1-8.

16. Sanga LA., et al. "Inter-pregnancy interval and associated adverse maternal outcomes among women who delivered at Kilimanjaro Christian Medical Centre in Tanzania, 2000-2015". PLoS ONE 15.2 (2020): e0228330.

17. WHO recommendations on antenatal care for a positive pregnancy experience.2017; World Health Organization Newborns: reducing mortality (2017).

18. Nsangamay T and Mash R. "How to improve the quality of care for women with postpartum hemorrhage at Onandjokwe Hospital, Namibia: quality improvement study". BMC Pregnancy and Childbirth (2019): 19-489.
19. Heemelaar S., et al. "Measuring maternal near-miss in a middle-income country: assessing the use of WHO and subSaharan Africa maternal near-miss criteria in Namibia". Global Health Action 12.1 (2019): 1646036.

20. Lilungulu A., et al. "Spectrum of maternal and perinatal outcomes among parturient women with preceding short inter pregnancy interval at Bugando Medical Centre, Tanzania". Maternal Health, Neonatology and Perinatology 1 (2015): 1.

21. Cecatti JG., et al. "The associations between inter-pregnancy interval and maternal and neonatal outcomes in Brazil". Maternal and Child Health Journal 12 (2008): 275-281.

22. Cunningham F., et al. "Hypertensive Disorders". In: Cunningham F, Leveno KJ, Bloom SL, et al. eds. Williams Obstetrics. $25^{\text {th }}$ edition. New York, NY: McGraw-Hill (2018): 1564-1166.

23. Rasmussen K. "Is there a causal relationship between iron deficiency or iron-deficiency anemia and weight at birth, length of gestation and perinatal mortality?" Journal of Nutrition 131 (2001): 590S-601S.

24. Milman N. "Oral iron prophylaxis in pregnancy: not too little and not too much". Journal of Pregnancy (2012): 514345.

25. Haider BA., et al. "Anemia, prenatal iron use, and risk of adverse pregnancy outcomes: systematic review and meta-analysis". British Medical Journal 346 (2013): f 3443.

26. WHO. Making health service adolescent friendly: Developing national quality standard for adolescent friendly health services. Geneva: WHO (2012).

27. Kalafa E and Thilaganatha B. "Cardiovascular origin of preeclampsia". Current Opinion Obstetrics and Gynecology 26.6 (2017): 383-389.

\section{Assets from publication with us}

- Prompt Acknowledgement after receiving the article

- Thorough Double blinded peer review

- Rapid Publication

- Issue of Publication Certificate

- High visibility of your Published work

Website: www.actascientific.com/

Submit Article: www.actascientific.com/submission.php

Email us: editor@actascientific.com

Contact us: +919182824667 